
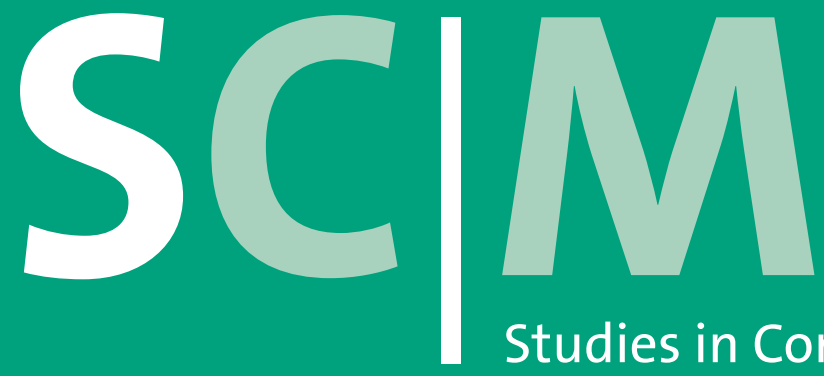

\title{
Studies in Communication | Media
}

\section{FULL PAPER}

Der Einfluss von Fernsehübertragungen von Mega-Events auf die Wahrnehmung des Gastgeberlandes

Eine Studie am Beispiel des Eurovision Song Contests in Aserbaidschan

The influence of televised mega-events on the perception of the hosting nation

A study through the example of the Eurovision Song Contest in Azerbaijan

Christiana Schallhorn 


\section{Kontakt}

Christiana Schallhorn, M.A.

Universität Würzburg

Medien- und Wirtschaftskommunikation

Institut Mensch-Computer-Medien

Hubland Campus Nord

Oswald-Külpe-Weg 82

D-97074 Würzburg

christiana.schallhorn(at)uni-wuerzburg.de 


\section{FULL PAPER}

\section{Der Einfluss von Fernsehübertragungen von Mega-Events auf die Wahrnehmung des Gastgeberlandes}

Eine Studie am Beispiel des Eurovision Song Contests in Aserbaidschan

\section{The influence of televised mega-events on the perception of the hosting nation \\ A study through the example of the Eurovision Song Contest in Azerbaijan}

\section{Christiana Schallhorn}

Zusammenfassung: Die Medienberichterstattung hat einen wesentlichen Anteil daran, wie wir die Realität wahrnehmen (Adoni \& Mane, 1984; Gerbner \& Gross, 1976). Insbesondere, wenn wir nicht über persönliche Erfahrungen verfügen, wie beispielweise mit anderen Ländern und Kulturen, bieten internationale Fernsehgroßereignisse informativ und unterhaltsam einen Einblick in andere Nationen (Gamson, Croteau, Hoynes, William, \& Sasson, 1992). Die Studie untersucht, wie das Wissen und Vorstellungen über Aserbaidschan durch das musikalische Mega-Event Eurovision Song (ESC) Contest beeinflusst werden. Es stellt sich die Frage, ob und wie sich die Wahrnehmung des Austragungslandes zwischen Personen ohne Vorerfahrungen, die die Sendung intensiv (Vielseher), teilweise (Teilseher) oder gar nicht (Nichtseher) verfolgt haben und Personen mit Vorerfahrungen voneinander unterscheidet. Die Ergebnisse der Online-Umfrage $(N=311)$ belegen, dass der Wissensvorsprung umso größer ist, je intensiver die Sendung verfolgt wurde. Es zeigt sich außerdem, dass sich die Vorstellungen der Personen, die den ESC nicht gesehen haben, signifikant von den anderen Gruppen (Vielseher und Teilseher ohne Vorerfahrung, Personen mit persönlichen Erfahrungen) unterscheiden. Diese Effekte zeigen das Potential und den Einfluss von Fernsehgroßereignissen auf.

Schlagwörter: Internationale Events, Wahrnehmung, Vorstellungen, Fernsehen, Länder

Abstract: Media play an important role in constructing reality (Adoni \& Mane, 1984; Gerbner \& Gross, 1976). Especially if we are unfamiliar with, for instances, other countries and cultures, international televised mega-events offer a closer, informative and entertaining look at other nations (Gamson, Croteau, Hoynes, William, \& Sasson, 1992). This study explores how knowledge and certain issues about Azerbaijan are influenced by the musical mega-event Eurovision Song Contest (ESC). The question is if and how the perception of the hosting nation differs between people without any contacts with Azerbaijan or Azerbaijani having followed intensively (heavy-viewers), partly (moderate-viewers) or not 
at all (non-viewers) the event and people possessing personal experiences. Results of the online questionnaire show $(N=311)$ that the more people had watched the event the more they knew about the country. Furthermore ideas of people having not seen the ESC differ significantly from the other groups (heavy- and moderate-viewers without personal experiences and persons with personal experiences). These outcomes demonstrate the power and influence of televised mega-events.

Keywords: international events, perception, images, television, nations

\section{Einleitung}

Medien haben einen großen Einfluss auf die Konstruktion sozialer Wirklichkeit (Adoni \& Mane, 1984, S. 323). Durch den technischen Fortschritt und die mediale Vernetzung haben nicht nur immer mehr Menschen Zugang zu Medienangeboten und Informationen, sondern für Rezipienten werden so auch Themenbereiche erfahrbar, zu denen sie in ihrem alltäglichen Leben keinen direkten Kontakt haben (Gamson, Croteau, Hoynes, William, \& Sasson, 1992, S. 386). Beispiele dafür sind die Außenpolitik, internationale Beziehungen und Globalisierung, ferne Länder und Kulturen. Wenn Personen nur begrenzte oder gar keine Möglichkeiten haben, sich persönlich ein Bild von einem Land oder einer Kultur zu machen, sind Massenmedien maßgeblich an der Entstehung von Nationenimages beteiligt (Bentele, 1995, S. 63). Bereits Lippmann (1929) verwies auf die Tatsache, dass wir vieles über die Welt erfahren bevor wir uns selbst ein Bild machen können (S. 90). Die Bedeutung der Medien in diesem Prozess der Realitätskonstruktion betont Luhmann (1996): „Was wir über unsere Gesellschaft, ja über die Welt, in der wir leben, wissen, wissen wir durch die Massenmedien “ (S. 9). Das Fernsehen nimmt dabei eine Sonderstellung ein, da „allen voran die Fernsehzuschauer, medial vermittelte Realität oftmals mit Primärerfahrung verwechseln" (Knieper, 2006, S. 53). Der Fernseher ist nach wie vor das Leitmedium unter den Medien, wenn es darum geht, sich über das Weltgeschehen zu informieren (Hasebrink \& Schmidt, 2012; Mende, Oehmichen, \& Schröter, 2012). Doch die Realität kann nie vollständig abgebildet werden (Niedhart, 1996, S. 81), vielmehr konstruiert jedes Mediensystem ein eigenes Bild von anderen Ländern (Bentele, 1995, S. 64). Verfügt der Rezipient bereits über Vorerfahrungen oder Vorwissen, werden die neuen Informationen mit bestehenden Vorstellungen abgeglichen und verknüpft. Werden Informationen aber erstmalig über die Medien aufgenommen, z. B. weil es sich um Themen handelt, die fern der eigenen Lebenswelt liegen, ist der Einfluss der medialen Darstellungen umso größer (Bilandzic, 2006).

\section{Wie das Fernsehen Vorstellungen über andere Länder und Kulturen prägt}

Die USA ist noch immer der weltweit größte Exporteur von Medieninhalten, Filmen und Fernsehprogrammen für andere Länder (Weimann, 2000, S. 247). Das wirkt sich auch auf die Vorstellungen von und über Amerika aus. Ein in den USA lebender Emigrant aus Israel beschrieb in diesem Zusammenhang: „Those movies were the real cause (...) they described life in America as a legend: big cars, rock 
music, dancing parties, plenty of everything (...). I believed that this is the way of life of everyone who lives in America" (ebd., 2000, S. 243). Dieses Beispiel belegt die kultivierende Wirkung des Fernsehens, bei denen wie von Gerbner und Kollegen angenommen (u. a. Gerbner \& Gross, 1976; Gerbner, Gross, Signorielli, \& Morgan 1980; Gerbner, Gross, Morgan, \& Signorielli, 1980, 1982, 1994), homogene Medienbotschaften, die über einen langen Zeitraum hinweg wiederholt werden, Vorstellungen von der Realität bei Rezipienten prägen. Wie stabil solche Vorstellungen sind, wies Hetsroni (2010) nach. Er stellte in einer Befragung in Israel fest, dass das jahrelang kultivierte positive Bild von Islands Wirtschaft bei den Israelis, die sehr viel allgemeine Zeitungen lasen, weiterhin bestehen blieb, als die Berichterstattung über Island aufgrund der Wirtschaftskrise negativer ausfiel (Hetsroni, 2010, S. 457).

Neben der alltäglichen Berichterstattung über das Weltgeschehen bieten Sportgroßereignisse wie die Olympischen Spielen oder Fußballweltmeisterschaften den Austragungsländern eine gute Möglichkeit, internationale, mediale Aufmerksamkeit zu erhalten (Giffard \& Rivenburgh, 2000; Ritchie \& Smith, 1991; Florek, Breitbarth, \& Conejo, 2008; Manzenreiter, 2010). Die Berichterstattung über die Gastgeberländer und Austragungsorte dauert zwar nur wenige Wochen an, ist aber in diesem Zeitraum dafür umso intensiver. Mehrfach konnte belegt werden, dass Besucher dieser Events die Austragungsländer danach deutlich positiver bewerten als vorher (Kim \& Morrison, 2005; Gibson, Qi, \& Zhang, 2008; Florek, Breitbarth, \& Conejo, 2008; Custódio \& Gouveia, 2007). Ob die Fernsehübertragung aber zu ähnlichen Effekten führt, wurde bisher weniger untersucht. Ritchie und Smith (1991) konnten zeigen, dass nach den Olympischen Winterspielen 1988 nicht nur das Wissen und der Bekanntheitsgrad des Austragungsorts Calgary in Europa und den USA drastisch anstieg, sondern noch ein Jahr später konstant geblieben war. Hede (2005) wies in einer Studie in Australien nach, dass die Übertragung der Olympischen Sommerspiele 2004 in Griechenland dessen Wahrnehmung als attraktives Reiseziel deutlich steigern konnte. Ausgehend von diesen Ergebnissen liegt die Annahme nahe, dass durch die Fernsehübertragung internationaler Medien-Events zumindest kurzzeitige Lerneffekte bei den Rezipienten nachzuweisen sind. Der Einfluss der Fernsehinhalte sollte zudem umso größer sein, wenn nur ein geringes Vorwissen und keine persönlichen Erfahrungen vorhanden sind (Adoni \& Mane, 1984, S. 331; Bilandzic, 2006, S. 347). Die vorliegende Studie beschäftigt sich daher mit der Frage, wie sich die Wahrnehmung eines weitgehend unbekannten Austragungslandes eines Mega-Events in Abhängigkeit vom Fernsehkonsum und persönlichen Erfahrungen bei Rezipienten unterscheidet.

\section{Mega-Events}

Der Begriff Mega-Event wird heutzutage sehr häufig verwendet, allerdings werden nicht alle so titulierten Ereignisse tatsächlich ihrer Bezeichnung gerecht (Klenk, 1999, S. 39). Weiß (2008) definiert Mega-Events als

einmalige oder wiederkehrende Ereignisse von zeitlich begrenzter Dauer, die auf Grund ihrer Einzigartigkeit eine global bedeutende mediale Aufmerksamkeit erzeugen und eine hohe Anziehungskraft auf Besucher weltweit zeigen. Die Vergabe folgt 
einem formalen Bewerbungsprozess. Sowohl für die Bewerbung als auch die Durchführung ist jeweils eine eigene Gesellschaft zu gründen, die durch enge Verflechtungen mit den vergebenden Rechteinhabern geprägt ist. (S. 40)

Diese Definition lässt sich durch den Aspekt der Internationalität erweitern. Demnach sollte eine Großveranstaltung in den internationalen Medien eine intensive Berichterstattung auslösen, um das Gastgeberland in möglichst vielen Ländern zu präsentieren. Dieses Ziel kann vor allem durch die Beteiligung möglichst vieler Nationen an einem Event erreicht werden (Klenk, 1999). Mega-Events lassen sich aber auch durch ihre Wirkung charakterisieren. Nach Katz, Dayan und Motyl (1981) bringen Mega-Events Menschen einer Nation zusammen, lassen sie zusammen das Ereignis im Fernsehen verfolgen und aufgrund des ungewissen Ausgangs mitfiebern und diskutieren. Mega-Events unterbrechen die Alltagsroutine von Rezipienten, der Öffentlichkeit und sogar der Fernsehanstalten (S. 48). Eine besondere Bedeutung haben sie aber für die Gastgeberländer. Internationale Fernsehgroßereignisse bieten Ländern die Gelegenheit, ihren Bekanntheitsgrad zu steigern, ihr Image zu verbessern, internationale Beziehungen zu stärken, für den Tourismus zu werben oder ausländische Investoren anzusprechen (Giffard \& Rivenburgh, 2000; Ritchie \& Smith, 1991; Florek, Breitbarth, \& Conejo, 2008; Manzenreiter, 2010). Insbesondere dann, wenn ein eher unbekanntes Land plötzlich umfangreiche mediale Aufmerksamkeit erhält, hat die Berichterstattung einen starken Einfluss auf seine Wahrnehmung (Zeng, Go, \& Kolmer, 2011). Das große mediale Interesse an den Events birgt aber auch die Gefahr, dass Schattenseiten eines Landes beleuchtet werden und in der öffentlichen Kritik stehen. Schließlich kann nicht kontrolliert werden, worüber und wie die Medien letztendlich berichten (Giffard \& Rivenburgh, 2000, S. 10). Neben Studien zu Mega-Events im Zusammenhang mit Sportgroßereignissen wie den Olympischen Spielen oder Fußballweltmeisterschaften werden nur vereinzelt die Wirkung anderer Events, wie den internationalen Weltausstellungen (Klenk, 1999; Roche, 2000) oder dem Eurovision Song Contest (Georgiou, 2008; Wolter, 2006) untersucht. Auf den Eurovision Song Contest (ESC) wird in folgendem Abschnitt näher eingegangen, weil er als Untersuchungsgegenstand dieser Studie herangezogen wird.

\section{Ein musikalisches Mega-Event: Der Eurovision Song Contest (ESC)}

\subsection{Der ESC - ein aktuelles Gemeinschaftsprojekt mit Tradition}

Seit 1956 wird der Eurovision Song Contest (ESC) jährlich von der European Broadcasting Union (EBU) veranstaltet und hat damit eine lange Tradition. Ursprünglich diente der musikalische Wettkampf jedoch als Anlass dafür, dass die europäischen Fernsehanstalten regelmäßig bei einem internationalen Event zusammenarbeiten (Wolther, 2010, S. 11). Inzwischen hat sich der ESC als „weltweit größte[r] internationale[r] Wettbewerb für populäre Musik“ (Wolther, 2005, S. 101) etabliert und ist weit mehr als nur ein von Fernsehanstalten inszeniertes Musikereignis. Doch kann er auch als Mega-Event bezeichnet werden? Zieht man die eingangs genannte Definition von Mega-Events heran, so erfüllt der ESC die 
Kriterien: Er findet jedes Jahr statt, erzeugt eine große - wenn auch nicht globale, aber dennoch mindestens europaweite - mediale Aufmerksamkeit. Scherer und Schlütz (2003) führen das darauf zurück, dass der ESC „per se einen hohen Nachrichtenwert hat, aber dessen mediale Aufbereitung zusätzlich berichtenswert ist" (S. 153). Viele Fans reisen zum Veranstaltungsort, aber auch die Übertragung im Fernsehen erfreut sich großer Beliebtheit. Im Jahr 2011 war der ESC mit 13,93 Millionen Zuschauern und einem Marktanteil von 49,4 Prozent die erfolgreichste Unterhaltungssendung in Deutschland. Die Musiksendung verwies Wetten, dass...? mit durchschnittlich 10,58 Millionen Zuschauern und einen Marktanteil von 33,8 Prozent auf den zweiten Platz (Zubayr \& Gerhard, 2012, S. 131). Der Eurovision Song Contest belegte mit der damals für Deutschland auftretenden Lena sogar den achten Platz der meistgesehenen Einzelsendungen des Jahres 2011 (ebd., S. 127). ${ }^{1}$ Allein diese Zahlen zeigen das große Interesse des Eurovision Song Contests in Deutschland auf.

\subsection{Der ESC - mehr als nur eine Musiksendung}

Doch hinter dem einstigen Wettbewerb um den besten Song Europas steckt inzwischen viel mehr. Der Eurovision Song Contest hat ein hohes national-kulturelles Repräsentationspotenzial (Wolter, 2008). Arntsen sieht im ESC „an illustration of process of globalisation and international trade in television formats" (Arntsen, 2005, S. 147). Auch Georgiou (2008) verweist auf die kulturübergreifende Bedeutung des ESCs und beschreibt ihn als „a unique case, for it so intensely brings together nation-centrism, transnational politics and the construction of identity and otherness" (S. 142). Die Teilnehmer des ESCs erzeugen durch ihre Auftritte bestimmte Bilder über das Land, das sie vertreten. Somit stellt der ESC ein „kulturelles Forum der nationalen Selbstdarstellung “ dar (Schweiger \& Brosius, 2003, S. 274). Die erzeugten Bilder und Vorstellungen bei den Rezipienten können auch über die Sendung hinaus bei den ZuschauerInnen bestehen bleiben (ebd. S. 142). Das heißt, auch Entwicklungen durch die EU-Erweiterung und Globalisierung spiegeln sich im ESC wieder: Er bringt verschiedenste Kulturen in einer Sendung zusammen, führt das gemeinsame Projekt der europäischen Fernsehanstalten fort und regt jedes Jahr aufs Neue zu Diskussionen über Teilnehmer und Wahlpräferenzen der Länder bei der Punktevergabe an (Schweiger \& Brosius, 2003; Yair, 2005, Ginsburgh \& Noury, 2008, Vallant, 2011). Wolther (2006) spricht nach einer Analyse von sieben Bedeutungsdimensionen des ESC. Dazu zählen die mediale Dimension, die musikalische Dimension, die musikökonomische Dimension, die politische Dimension, die national-kulturelle Dimension, die nationalökonomische Dimension und die kompetitorische Dimension. Relevant im Zusammenhang mit der Wahrnehmung des ESC als Mega-Event sind insbesondere die national-kulturelle und die nationalökonomische Dimensionen. Sie werden nachfolgend näher beschrieben.

1 Die vorderen Plätze der meistgesehenen Einzelsendungen belegten 2011 die Spiele der FrauenFußball-WM, der Boxkampf Klitschko vs. Haye und Gottschalks letzte „Wetten, dass?“-Sendung 


\subsection{Der ESC - Chancen für Kultur und Wirtschaft}

Die national-kulturelle Dimension umfasst vor allem die nationale Identität und die kulturellen Besonderheiten eines Landes. Trotz der inzwischen vorwiegend englischsprachigen Vorträge versuchen die Interpreten folkloristische oder kulturspezifische Elemente ihrer Heimatländer in ihren Auftritten zu integrieren, um etwas Landestypisches zu präsentieren (Wolther, 2006, S. 127). Wolther (2008) sieht in der „national-kulturellen Identifikation mit dem Beitrag des eigenen Landes den Schlüssel für den anhalten Erfolg des Wettbewerbs“ (ebd., S. 111). Daher ist es naheliegend, dass der ESC insbesondere für kleine, weniger bekannte oder krisengeprägte Länder von enormer Bedeutung ist, da er das Gefühl der Zusammengehörigkeit stärkt (Wolther, 2006, S. 137). Gleichzeitig sind diese Länder besonders an der Austragung des Eurovision Song Contests interessiert, da die Sendung eine ideale Möglichkeit bietet, sich in einem bestimmten Licht einem internationalen Millionenpublikum und potentiellen Investoren zu präsentieren. So stellte sich Norwegen 1996 als Land der Gegensätze dar, das einerseits durch die Wikinger auf seine ursprünglichen und authentischen Wurzeln verwies, andererseits aber auf sich als vielversprechender Industriestandort mit entstehenden Arbeitsplätzen und Investitionsmöglichkeiten aufmerksam machte (Arntsen, 2005, S. 151). Inzwischen werden nicht nur die Umbauphasen während der Sendung für Impressionen aus dem Gastgeberland genutzt, sondern die zusätzlich eingeführten Halbfinales im Jahr 2004 tragen dazu bei, dass noch mehr Raum für die Berichterstattung und mediale Inszenierung rund um den musikalischen Wettbewerb bleibt. An dieser Stelle schließt sich die nationalökonomische Dimension an. Es hat sich mehrfach bestätigt, dass sich die Ausrichtung des Eurovision Song Contests lohnt: Estland und Lettland präsentierten sich als sehenswerte Reiseziele und warben für sich als attraktive Standorte für Wissenschaft und Industrie vor ihren EU-Beitritten. Irland, das drei Jahre in Folge (1992-94) den ESC gewann und wiederholt als Gastgeber glänzte, verzeichnete eine hohe Zunahme an Besucherzahlen (Wolther, 2006, S. 141). Somit ist die Veranstaltung „besonders für kleine und relativ unbekannte Länder (...) mit ihrer weiten öffentlichen Beachtung in Europa von immenser Bedeutung " (Schweiger \& Brosius, 2003, S. 274). Doch welche Eindrücke hinterließ das musikalische Mega-Event Eurovision Song Contest 2012 in Aserbaidschan? Interessant wird diese Fragestellung vor allem vor dem Hintergrund, dass Aserbaidschan außerhalb des ESCs kaum in den deutschen Medien erwähnt wird. Das heißt, es ist für die deutsche Bevölkerung ein weitgehend unbekanntes Land - wodurch anzunehmen ist, dass die Berichterstattung die Vorstellungen über Aserbaidschan bei den deutschen Rezipienten prägt. Wie unterscheidet sich folglich die Wahrnehmung Aserbaidschans zwischen Personen, die die Sendung nicht gesehen haben, zu denen die sie teilweise oder intensiv verfolgt haben? Und wie beurteilen wiederum Personen, die bereits persönliche Erfahrungen mit Aserbaidschanern oder in Aserbaidschan gemacht haben das Land im Vergleich zu den anderen Befragten ohne persönliche Primärerfahrung? Diesen Fragen wird in vorliegender Studie nachgegangen. 


\section{Zwischenfazit und Hypothesen}

Mega-Events lassen sich zusammengefasst als zeitlich begrenzte Großereignisse beschreiben, die durch die Teilnahme mehrerer Nationen große mediale Aufmerksamkeit in vielen Ländern erzeugen und ein großes internationales Publikum erreichen (Weiß, 2008; Klenk, 1999; Katz, Dayan, \& Motyl, 1981). Mega-Events wirken sich auf verschiedenen Dimensionen aus (vgl. Wolter, 2006) und beeinflussen durch die intensive Berichterstattung über das Event auch die Wahrnehmung des Austragungslandes. Der Eurovision Song Contest kann damit als musikalisches Mega-Event bezeichnet werden. Aserbaidschan fand vor dem Eurovision Song Contest kaum Beachtung in den deutschen Medien. Das heißt, bei Personen ohne persönliche Vorerfahrungen sollte der Rezeptionsumfang der Sendung einen großen Einfluss auf das Wissen über Aserbaidschan haben. Anzunehmen ist deshalb:

H1: Personen, die den Eurovision Song Contest intensiv verfolgt haben (Vielseher), wissen mehr über Aserbaidschan als Personen, die den ESC nur teilweise (Teilseher) oder gar nicht (Nichtseher) verfolgt haben.

Außerdem wird vermutet, dass Personen, die Aserbaidschaner persönlich kennen oder schon in Aserbaidschan waren, bereits Wissen über Aserbaidschan erworben haben. Deshalb soll auch überprüft werden, wie stark sich Befragte mit persönlichen Primärerfahrungen von den anderen drei Gruppen ohne Vorerfahrungen unterscheiden. Da keine Aussagen darüber gemacht werden können, wie sehr persönliche Erfahrungen das Wissen beeinflussen, wird folgende Frage formuliert:

F1: Wie unterscheidet sich das Wissen über Aserbaidschan von Personen mit persönlichen Erfahrungen zu Aserbaidschan bzw. Aserbaidschanern zu Personen ohne Vorerfahrungen, die den ESC intensiv (Vielseher), teilweise (Teilseher) oder gar nicht (Nichtseher) verfolgt haben?

Katz et al. (1981) kritisieren, dass Gastgeberländer bei großen Events meist sehr unkritisch von den Journalisten dargestellt werden und die Medien stattdessen versuchen, die Zuschauer durch positive Emotionen mitzureißen (S. 48). Und (natürlich) erkennen die Austragungsländer die Möglichkeit, sich durch ein MegaEvent international zu vermarkten und die positiven Seiten ihres Landes in den Mittelpunkt der Berichterstattung zu rücken (Giffard \& Rivenburgh, 2000; Ritchie \& Smith, 1991; Florek, Breitbarth, \& Conejo, 2008; Manzenreiter, 2010). Während der Sendung wurden beeindruckende, schöne Bilder und Filmsequenzen von Aserbaidschan eingeblendet. Jedoch verwies der Sprecher kritisch auf die politische Lage: „Wären da nicht die Schatten, die das Bild trügen. Korruptionsvorwürfe, Menschenrechtsverletzungen, Einschränkung von Pressefreiheit, Gänge- 
lung der politischen Opposition. “ 2 Anke Engelke mahnt vor der Punktevergabe aus Deutschland: „Tonight nobody could vote for their own country but it is good to be able to vote and it is good to have a choice. Good luck on your journey Azerbaijan, Europe is watching you. " Bereits im Vorfeld der Finalsendung wurde die Umsetzung der Demokratie in Aserbaidschan heftig in den Medien und von Menschenrechtlern verschiedener Länder kritisiert. So warf die Menschenrechtsorganisation Amnesty International den ESC-Organisatoren vor, Menschrechtsverstöße zu ignorieren und damit „den Behörden unbeschränkte Vollmacht [zu geben], weiterhin abweichende Meinungen mit Gewalt zu unterdrücken, ohne Konsequenzen fürchten zu müssen “. Dementsprechend sollte sich die Wahrnehmung von Aserbaidschan in der deutschen Bevölkerung in Abhängigkeit davon, ob die Übertragungen des ESCs intensiv, teilweise oder gar nicht verfolgt wurden, bei Personen ohne persönliche Vorerfahrungen unterscheiden.

H2: Personen, die den ESC intensiv (Vielseher), teilweise (Teilseher) oder nicht gesehen haben (Nichtseher) unterscheiden sich in ihrer Wahrnehmung voneinander.

Hypothese zwei soll durch vier Teilhypothesen differenziert betrachtet werden. Es geht darum, wie die Befragten die Attraktivität, Stabilität, Industrialisierung und Umsetzung der Demokratie von Aserbaidschan bewerten.

H2a Vielseher, Teilseher und Nichtseher unterscheiden sich in der Bewertung der Attraktivität von Aserbaidschan.

$H 2 b$ Vielseher, Teilseher und Nichtseher unterscheiden sich in der Bewertung der Stabilität von Aserbaidschan.

$\mathrm{H} 2 \mathrm{c}$ Vielseher, Teilseher und Nichtseher unterscheiden sich in der Bewertung der Industrialisierung von Aserbaidschan.

H2d Vielseher, Teilseher und Nichtseher unterscheiden sich in der Bewertung der Umsetzung der Demokratie in Aserbaidschan.

2 Kurz vor der Abstimmung während der Sendung erfolgt nach dem Schnelldurchlauf aller Teilnehmer ein Video mit vielen Bildern Aserbaidschans und folgender Kommentierung, die ebenfalls zur Erstellung des Fragebogens herangezogen wurde: „Hier noch mal geballte Sammlung der schönen Bilder aus Aserbaidschan und seiner schönen Hauptstadt Baku. Aserbaidschan ist ein spannendes, oft beeindruckendes Land. Und Baku ist seine faszinierende pulsierende Metropole mit einer von der UNESCO zum Weltkulturerbe erklärten Altstadt. Man spürt den Reichtum, der durch Erdöl und Erdgas erwirtschaftet wurde. Baku wirkt manchmal amerikanisch, manchmal europäisch elegant, manchmal orientalisch. Eine Mischung aus Nizza, Jerusalem und Dubai. Doch der Reichtum scheint auch der Allgemeinheit zugute zu kommen, in Form von Museen, Theatern, Konzerthäusern mit spektakulärer Architektur. Mit Parks, üppigen Uferpromenaden und Erholungsgebieten. Wären da nicht die Schatten, die das Bild trügen. Korruptionsvorwürfe, Menschenrechtsverletzungen, Einschränkung von Pressefreiheit, Gängelung der politischen Opposition. Man muss sich immer klarmachen, wo Aserbaidschan, die frühere Sowjet-Republik liegt. Umgeben von der Osttürkei, Russland, Armenien, Georgien und dem Nachbarn Iran. Zwischen Europa und Asien, zwischen Ost und West. Aber Aserbaidschan möchte sich nach Westen orientieren und ist Mitglied im Europarat geworden. Und damals hat die Regierung demokratische Reformen versprochen. Aber ob diese Reformen nun auch realisiert werden, steht immer noch in den Sternen. " (Eurovision Song Contest am 26.05.2012; Zeit des Videostreams: 2:08:20 - 2:10:35; Executive Producer: A. Karimli) 
Zudem soll, wie schon in der Forschungsfrage F1, ein Vergleich der Vielseher, Teilseher und Nichtseher ohne Vorerfahrungen mit Personen mit persönlichen Erfahrungen mit Aserbaidschan/-ern durchgeführt werden. Da nur unzureichende Informationen vorliegen, ob Personen mit persönlichen Beziehungen zu Aserbaidschan eher die Vorstellungen der Vielseher, Teilseher oder Nichtseher teilen, soll erneut statt einer Hypothese eine Frage formuliert werden:

F2: Wie unterscheiden sich die Vorstellungen über Aserbaidschan von Personen mit persönlichen Erfahrungen zu Aserbaidschan bzw. Aserbaidschanern von Vielsehern, Teil-sehern und Nichtsehern ohne persönliche Erfahrungen hinsichtlich Attraktivität, Stabilität, Industrialisierung und Umsetzung der Demokratie?

\section{Methode}

\subsection{Studiendesign}

Direkt im Anschluss an die Fernsehübertragung des Eurovision Song Contests wurde ein Online-Fragebogen freigeschaltet. Dieser wurde zum einen auf einschlägigen Foren zum ESC, einer Homepage eines Fanclubs zum ESC, dem deutschen Internetauftritt zum ESC, sowie auf sozialen Netzwerken verlinkt, um möglichst ESC-affine Personen und damit jene, die den ESC intensiv verfolgt haben, anzusprechen. Zusätzlich sollten Personen rekrutiert werden, die weniger Interesse am ESC haben und die Sendung teilweise oder nicht geschaut haben. Deshalb wurde der Link auch auf verschiedenen Internetseiten und -foren ohne Musik-Bezug platziert. Der Fragebogen war anschließend für zehn Tage online (27.05.2012-06.05.2012).

\subsection{Messung}

Der Fragebogen setzte sich aus mehreren Teilen zusammen. Im Folgenden werden nur die für die Auswertung relevanten Teile vorgestellt. Nach einer Einstiegsfrage folgten die Itembatterien zur Einschätzung von Aserbaidschan vor den Fragen zum ESC, um ein (ohnehin schon naheliegendes) Framing durch den ESC zu vermeiden. Die 17 Items zu Vorstellungen über Aserbaidschan wurden auf einer 7-stufigen Likertskala abgefragt, bei der die Befragten die Aussagen zwischen „trifft überhaupt nicht zu“ (1) und „trifft voll und ganz zu“ (7) beurteilen sollten. Die Items ließen sich zu vier Indices zusammenfassen: Demokratie, Industrialisierung, Stabilität und Attraktivität des Landes.

Der Index „Demokratie“ $(\alpha=0.90)$ setzte sich aus den vier Items zusammen: Aserbaidschan [1] achtet die Menschenrechte, [2] schützt die Meinungsfreiheit [3] ist demokratisch und [4] wird gut regiert. Der Index „Industrialisierung des Landes" $(\alpha=0.83)$ wurde durch die drei Items: Aserbaidschan ist [1] hochindustrialisiert, [2] hat ein gut entwickeltes Wirtschaftssystem und [3] ist eine High-Tech-Gesellschaft) abgefragt. Der Index „Stabilität des Landes“ ( $\alpha=0.74)$ enthielt die drei Items: Aserbaidschan [1] hat einen hohen Lebensstandard, [2] ist 
stabil und friedlich und [3] hat ein gutes Sozialsystem ${ }^{3}$. Den größten Index bildete die „Attraktivität des Land“ ( $\alpha=0.89)$ mit den sieben Items: Aserbaidschan [1] hat viele historische Bauwerke und Plätze, [2] begeistert in Großstädten mit innovativer Architektur, [3] hat attraktive, moderne Großstädte, [4] bietet ein vielseitiges Freizeitangebot, [5] beeindruckt mit modernen Skylines in Metropolen, [6] hat herrliche Urlaubs- und Erholungsgebiete, [7] zeichnet sich durch seine Kultur im Bereich Musik und Theater aus.

Anschließend gab es vier Wissensfragen über Aserbaidschan, wobei unter vier vorgegebenen Antwortmöglichkeiten nur eine Richtige und die Option „,ich weiß es nicht" gegeben war. Die Fragen lauteten: [1] Was ist die Hauptstadt von Aserbaidschan ${ }^{4}[2]$ Wie heißt der aktuelle Regierungschef von Aserbaidschan?5 [3] Welche Sprache ist die Landessprache von Aserbaidschan? ${ }^{6}$ [4] Welches Land grenzt nicht an Aserbaidschan? ${ }^{7}$

Es folgten Fragen zum Rezeptionsumfang des ESC, um bei der Auswertung zwischen „Vielsehern“, „Teilsehern“ und „Nichtsehern“ unterscheiden zu können. Zunächst wurde dichotom abgefragt, welche Sendungen des ESC angeschaut wurden: den deutschen Vorentscheid („Unser Star für Baku“), mindestens eines der zwei Halbfinales, das Wort zum Sonntag, ${ }^{8}$ das Finale. Wurde angegeben, dass das Finale angeschaut wurde, erfolgte eine weitere Frage zu den Programmteilen der Finalshow: „Was haben Sie sich alles angesehen? Und wie viel von der Sendezeit bzw. der Auftritte haben Sie sich ungefähr angeschaut?" Antworten waren auf einer 7-er Skala von gar nicht (1) bis komplett (7) möglich. Zu den drei Items gehörten [1] Auftritt von Roman Lob, unserem Star für Baku, [2] Auftritte anderer Länder bei Eurovision Song Contest und [3] Punktevergabe der Länder. Bei den demografischen Daten wurde neben Angaben zu Alter, Geschlecht und Bildung zusätzlich abgefragt, ob der Teilnehmer Aserbaidschaner persönlich kennt oder bereits in Aserbaidschan war.

\subsection{Stichprobe}

Der Datensatz enthält 311 gültige Fälle bei einer Beendigungsquote von 53 Prozent. 67 Prozent der Befragten sind Männer, 33 Prozent Frauen. Der Altersdurchschnitt liegt bei 32 Jahren $(S D=11,1$ Jahre) und $85 \%$ haben mindestens das Abitur erreicht. 15 Prozent der Teilnehmer kennen Aserbaidschaner persönlich und 6 Prozent sind schon einmal in Aserbaidschan gewesen. Die Stichprobe kann damit nicht als repräsentativ angesehen werden, da zum einen ein deutlicher Bildungs- und Geschlechterbias im Vergleich zur Gesamtbevölkerung vorliegt.

3 Die Formulierung der Items für die Indices Demokratie, Industrialisierung und Stabilität erfolgte in Anlehnung an Pappu, Quester und Cooksey (2007) sowie Martin und Eroglu (1993).

4 Lösungsvorschläge: Baku, Gändschä, Sumgajit, Mingetschewir.

5 Lösungsvorschläge: Fərhad Vəliyev, Idrak Abbasow, Ilham Alijew, Husein Arablinski.

6 Lösungsvorschläge: Aserbaidschanisch, Russisch, Türkisch, Armenisch.

7 Lösungsvorschläge: Georgien, Türkei, Armenien, Syrien.

8 Das Wort zum Sonntag wurde direkt vor dem ESC-Finale gesendet und widmete sich kritisch den Zuständen in Aserbaidschan. Kritisiert wurden die eingeschränkte Meinungsfreiheit und die Gefahr für Journalisten, die über Missstände berichten. 
Zum anderen weist die mäßige Beendigungsquote auf eine hohe Selbstselektion der Teilnehmenden hin, was bei Online-Umfragen ohnehin oft problematisch ist (Hauptmanns \& Lander, 2003).

Die Befragten wurden zunächst in die vier Gruppen „persönliche Erfahrung“, „Vielseher", „Teilseher" und „Nichtseher" wie folgt eingeteilt:

Personen, die persönliche Erfahrungen mit Aserbaidschanern und/oder in Aserbaidschan angaben, wurden als Gruppe mit „persönlicher Erfahrung“ ( $n=52)$ definiert, unabhängig vom Umfang der Rezeption von ESC-Inhalten. ${ }^{9}$

Personen, die weder den Vorentscheid, noch ein Halbfinale und auch nicht das Finale angeschaut haben, keine Aserbaidschaner persönlich kennen und auch noch nie in Aserbaidschan waren, wurden als „Nichtseher“ $(n=45)$ klassifiziert. Ziel war es, auf diese Weise eine Extremgruppe zu bilden, die Informationen über den ESC in Aserbaidschan höchstens marginal aus der alltäglichen Berichterstattung oder Gesprächen mitbekommen hat.

Der Gruppe „Vielseher“ ( $n=53)$ wurden alle TeilnehmerInnen zugeordnet, die den Vorentscheid, mindestens ein Halbfinale (beide dichotom abgefragt), das Wort zum Sonntag sowie das Finale komplett (7-er Skala, bei allen Items der Programmteile sieben) gesehen haben und dazu keine persönlichen Erfahrungen mit Aserbaidschanern bzw. Aserbaidschan hatten (dichotom abgefragt). Diese Gruppe stellt das entgegengesetzte Extrem zu den Nichtsehern dar. Vielseher haben laut Kriterien seit Beginn des Vorentscheids Mitte Januar 2012 bis hin zu den Halbfinales und des Finales Ende Mai 2012 deutlich intensiver Informationen rund um den ESC rezipiert und aufgenommen. Sie haben alle offiziellen ESC-Sendungen über einen Zeitraum von vier Monaten gesehen.

Die Gruppe der „Teilseher“ ( $n=53)$ bildeten Personen, die etwa die Hälfte des ESC-Finales gesehen haben ${ }^{10}$, das Halbfinale oder der Vorentscheid nicht gesehen wurde und keine persönlichen Vorerfahrungen zu Aserbaidschan/-ern vorhanden waren. Durch die Auswahlkriterien sollte die Gruppe in etwa genau die „Mitte“ zwischen Nichtsehern und Vielsehern bilden.

Alle anderen Fälle wurden von der Analyse ausgeschlossen ${ }^{11}$, wodurch sich die Anzahl der Datensätze auf $n=204$ reduzierte, aber immer noch eine zufriedenstellende Teilnehmerzahl umfasste, die ausgeglichen über die vier Gruppen verteilt war.

Vielseher, Teilseher und Nichtseher haben gemeinsam, dass sie die Primärerfahrung mit Aserbaidschan durch die Medien gemacht haben. Daher sollten sich die

914 Befragte, gaben an schon in Aserbaidschan gewesen zu sein und persönlich Aserbaidschaner zu kennen. Fünf Befragte waren bereits in Aserbaidschan, kennen aber keine Aserbaidschaner persönlich. 33 Personen kennen Aserbaidschaner persönlich, waren aber noch nie in Aserbaidschan. 42 von 52 Befragte dieser Gruppe gaben an, den ESC komplett gesehen zu haben.

10 Auswahlkriterium war das Item über den Sehumfang der Auftritte anderer Länder bei Eurovision. Wurde 4 oder weniger angegeben (entspricht dann etwa die Hälfte der Sendung oder weniger), wurde die Person als Teilseher eingeordnet.

11 Ausgeschlossen wurden jene, die die Sendung zu weniger als 50 Prozent gesehen haben oder mehr als 50 Prozent angeschaut haben und zusätzlich die Vorentscheide oder Halbfinals verfolgt haben. Ziel der Einteilung war die Bildung zweier Extremgruppen (Nichtseher und Vielseher) und einer dritten Gruppe (Teilseher), die etwa die Hälfte der Sendung gesehen hat - also möglichst genau zwischen den Extremgruppen einzuordnen ist. 
Gruppen in ihrer Wahrnehmung Aserbaidschans unterscheiden, wie in Hypothese eins und zwei postuliert.

\section{Ergebnisse}

Das Wissen über Aserbaidschan variiert stark zwischen den Gruppen. Während die Hauptstadt Baku von allen Gruppen mit großer Mehrheit genannt werden konnte, zeigten sich bei den anderen Fragen große Wissensunterschiede. Je mehr der ESC verfolgt wurde, desto größer ist auch das Wissen über Aserbaidschan in den abgefragten Kategorien. So heben sich die Vielseher deutlich von den Teilsehern und Nichtsehern ab. Große Unterschiede werden insbesondere bei der Frage zum Regierungschef und der Landessprache sichtbar, bei denen nur jeder fünfte Nichtseher, jeder zweite bis dritte Teilseher, aber fast alle Vielseher die richtige Lösung kannten. Hypothese 1, in der angenommen wird, dass Personen, die den Eurovision Song Contest intensiv verfolgt haben (Vielseher), mehr über Aserbaidschan wissen als Personen, die den ESC nur teilweise (Teilseher) oder gar nicht (Nichtseher) verfolgt haben, kann bestätigt werden. Ein Vergleich der Gruppe mit persönlichen Vorerfahrungen zu den anderen Gruppen ohne persönliche Erfahrungen (F1) macht deutliche Unterschiede zu den Teil- und Nichtsehern sichtbar. Persönliche Erfahrungen ebenso wie eine intensive Verfolgung der Sendung führen zu einem hohen Wissensvorsprung gegenüber Nicht- und Teilseher (Abb. 1).

\section{Abbildung 1: Richtig gegebene Antworten in Prozent nach Gruppen.}

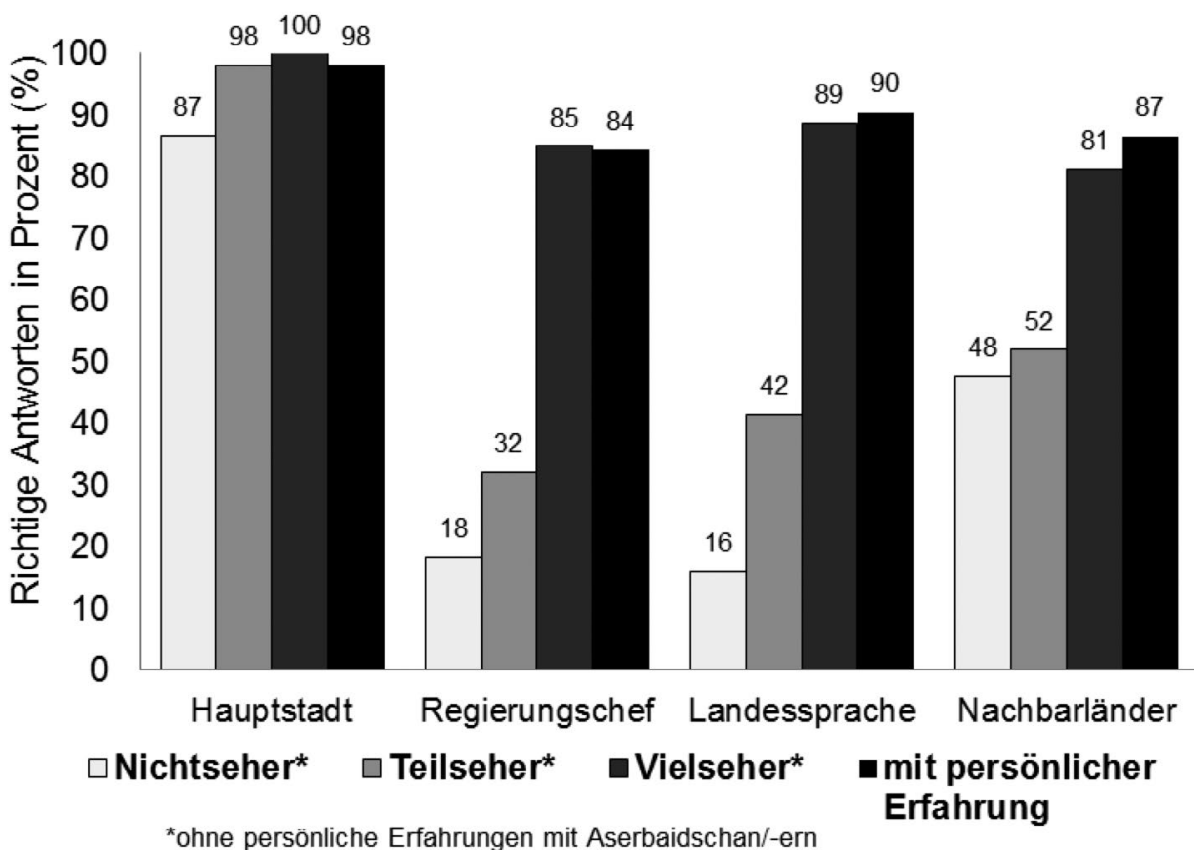


Im Folgenden soll die Frage geklärt werden, wie Personengruppen die Attraktivität, Stabilität, Industrialisierung und die Umsetzung der Demokratie bewerten. Dazu wurden einfaktorielle Varianzanalysen (ANOVA) sowie Post-Hoc-Tests nach Bonferroni durchgeführt, um zu untersuchen, welche Gruppen sich signifikant unterscheiden.

Attraktivität. Die ANOVA weist zunächst auf signifikante Gruppenunterschiede hin $\left(F(3,199)=12.13, p \leq .001, \eta^{2}=.16\right)$. Vielseher $(M=4.72, S D=.95, n=53)$ bewerten Aserbaidschan am attraktivsten und damit noch etwas besser als Personen mit persönlicher Erfahrung $(M=4.69, S D=1.16, n=52)$. Teilseher $(M=4.30$, $S D=1.16, n=53$ ) bewerten Aserbaidschan weniger attraktiv als Vielseher, aber dennoch deutlich positiver als Nichtseher $(M=3.55, S D=1.03, n=45)$. Der PostHoc-Test nach Bonferroni zeigt, dass diese Unterschiede der Bewertung der Attraktivität nur signifikant zwischen den Nichtsehern im Vergleich zu den drei anderen Gruppen sind (zu den Teilsehern $p<.05$; zu Vielsehern und Gruppe mit persönlichen Erfahrungen $p<.001$ ). Das heißt, durch das Sehen der Sendungen/ Sendungsteile zum ESC wird das Land signifikant attraktiver wahrgenommen als wenn die Sendungen nicht gesehen wurden. Das wird auch durch die große Effektstärke von 16 Prozent Varianzaufklärung unterstrichen. Hypothese 2a kann dahingehend bestätigt werden, dass sich Nichtseher signifikant von allen anderen Sehergruppen in der Bewertung der Attraktivität unterscheiden.

Stabilität. Bei der Bewertung der Stabilität Aserbaidschans gibt es signifikante Gruppenunterschiede $\left(F(3,198)=3.38, p \leq .05, \eta^{2}=.05\right)$. Personen mit persönlicher Vorerfahrung $(M=3.09, S D=1.09, n=52)$ erreichen beim Stabilitätsindex den höchsten Mittelwert, gefolgt von den Vielsehern ( $M=2.94, S D=.96, n=53)$. Damit liegen diese beiden Gruppen nah beieinander und unterscheiden sich nicht signifikant. Nichtseher $(M=2.63, S D=1.03, n=44)$ bewerten die Stabilität noch stärker als Teilseher $(M=2.52, S D=1.09, n=53)$, aber nicht signifikant. Statistisch signifikant unterscheiden sich bei diesem Index die Teilseher von den Personen mit persönlichen Erfahrungen $(p<.05)$. Denkbar wäre, dass Teilseher zufällig jene Programmteile gesehen haben, die eine negativere Wahrnehmung beispielsweise des Lebensstandards oder Sozialsystems erzeugt haben, während sich diese Vorstellungen bei Vielsehern durch umfangreichere Informationsaufnahme relativiert haben. Wie in Hypothese $2 \mathrm{~b}$ angenommen, bestätigen sich Unterschiede in der Bewertung der Stabilität, die jedoch nur zwischen Teilsehern und Personen mit Erfahrungen signifikant sind.

Industrialisierung. Die Varianzen in der Bewertung der Industrialisierung $(F(3$, 199) $\left.=7.65, p \leq .001, \eta^{2}=.10\right)$ können mit zehn Prozent durch die Gruppenbildung erklärt werden. Nichtseher $(M=2.77, S D=1.82, n=45)$ bewerten das Land weniger industrialisiert. Im Gegensatz dazu stehen die anderen drei Gruppen: Vielseher des ESCs $(M=3.97, S D=1.29, n=53)$ bewerten die Industrialisierung positiver als Personen mit persönlichen Erfahrungen $(M=3.65, S D=1.18, n=52)$ und Teilseher $(M=3.57, S D=1.37, n=53)$. Der Post-Hoc-Test nach Bonferroni bestätigt, dass erneut der Unterschied zwischen den Nichtsehern zu den anderen Gruppen signifikant ist und sich mit zunehmender Rezeption des ESCs vergrößert. Die mittlere Differenz zwischen Nichtsehern zu Teilsehern beträgt -.79 ( $p<.05)$, zu Vielsehern $-1.19(p<.001)$ und Personen mit persönlichen Erfahrungen -.88 ( $p<.01)$. Folglich 
hat auch für die Bewertung der Industrialisierung das Sehen der Sendungen einen erheblichen, signifikanten Einfluss, wobei die Bewertung bei steigendem Rezeptionsumfang positiver wird. Signifikante Unterschiede zwischen Nichtsehern und den anderen Sehergruppen können gemäß $\mathrm{H} 2 \mathrm{c}$ bestätigt werden.

Umsetzung der Demokratie. Die Zustimmung zu diesem Index variiert ebenfalls stark in Abhängigkeit von den Gruppen $\left(F(3,198)=8.14, p \leq .001, \eta^{2}=.11\right)$. Vielseher $(M=1.47, S D=0.51, n=53)$ stehen der Umsetzung der Demokratie am kritischsten gegenüber. Teilseher $(M=1.71, S D=.83, n=53)$ und Personen mit persönlichen Erfahrungen $(M=1.78, S D=1.00, n=52)$ bewerten die Umsetzung der Demokratie auch sehr kritisch, unterscheiden sich aber kaum voneinander. Nichtseher $(M=2.37, S D=1.25, n=44)$ weichen in ihrer Bewertung erneut stark und signifikant von den anderen Gruppen ab (zu den: Vielsehern $p<.001$, Personen mit persönlichen Erfahrungen $p<.01$, Nichtsehern $p<.05$; Post-Hoc-Test nach Bonferroni). Hypothese $2 \mathrm{~d}$ kann bestätigt werden. Je mehr die Sendung verfolgt wurde, desto kritischer wird die Umsetzung der Demokratie in Aserbaidschan bewertet, wobei die Unterschiede zwischen Nichtsehern und den anderen Gruppen signifikant sind.

Um die Frage zu beantworten (F2), wie sich die Wahrnehmung Aserbaidschans von Personen mit persönlichen Erfahrungen zu Aserbaidschan bzw. Aserbaidschanern zu Vielsehern, Teilsehern und Nichtsehern unterscheidet, lassen sich folgende Aussagen treffen: Zum einen unterscheiden sich Personen mit persönlichen Erfahrungen nicht signifikant von Personen, die die Sendung sehr intensiv verfolgt haben. Persönliche Erfahrungen führen aber dazu, dass Aserbaidschan stabiler bewertet wird als von den anderen Gruppen, wobei jedoch nur der Unterschied zu den Teilsehern signifikant ist. Weitere signifikante Unterschiede finden sich im Vergleich zu den Nichtsehern bei der Bewertung der Attraktivität und Demokratie: Personen mit persönlichen Erfahrungen bewerten Aserbaidschan attraktiver, die Umsetzung der Demokratie aber kritischer als Nichtseher (Abb. 2). 


\section{Abbildung 2: Bewertung Aserbaidschans nach Personengruppen.}

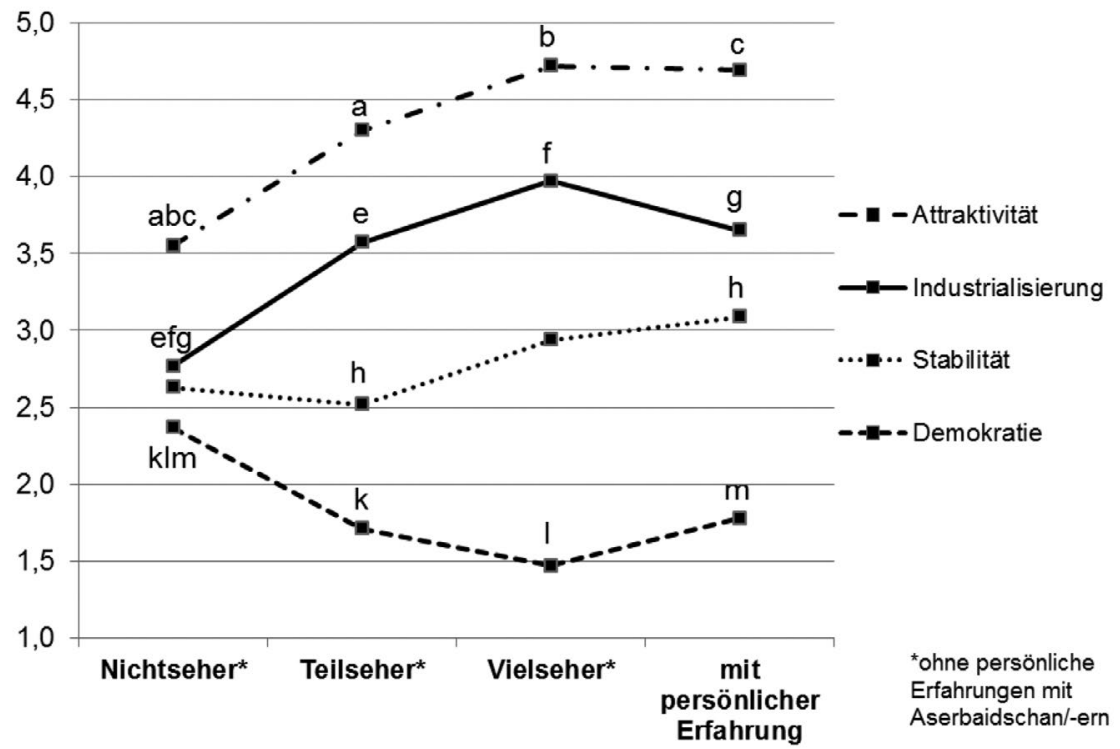

Anmerkung: Mittelwerte mit dem gleichen Kleinbuchstaben unterscheiden sich signifikant. (Bonferroni-korrigierte Post-hoc-Tests, $\mathrm{p}<.05$ )

Zusammenfassend lassen sich drei wesentliche Erkenntnisse aus der Studie ableiten: Erstens: Wie zu erwarten war, wissen Vielseher und Personen, die einen persönlichen Bezug zu Aserbaidschan/-ern haben, mehr als Teilseher und Nichtseher über das Land. Die Ergebnisse zur ersten Hypothese können als Beleg dafür gesehen werden, wie wenig über Aserbaidschan ohne Vorerfahrungen und ohne das Wissen aus den Sendungen zum ESC bekannt ist. Zweitens: Die Bewertung der Attraktivität, Industrialisierung und Demokratie von Aserbaidschan unterscheidet sich zwischen Personen mit persönlichen Erfahrungen und Personen, die den ESC teilweise oder intensiv verfolgt haben, nicht signifikant voneinander. Drittens: Die Gruppe der Nichtseher unterscheidet sich signifikant in den Bewertungen der Indices Attraktivität, Industrialisierung und Umsetzung der Demokratie von allen anderen Gruppen. Auch das kann als ein Indiz für den Einfluss der Sendung auf die Vorstellungen, die beim Rezipienten geprägt werden, gesehen werden. Die Ergebnisse sollen im folgenden Abschnitt diskutiert werden.

\section{Diskussion}

Die Ergebnisse spiegeln einen deutlichen Einfluss der Medien wieder. Die geringe Bekanntheit Aserbaidschans und das unterschiedlich starke Interesse am Eurovision Song Contest ermöglichte Gruppenvergleiche zwischen Nichtsehern, Teilsehern, Vielsehern und Personen mit persönlichen Erfahrungen. Am Beispiel des 
Mega-Events Eurovision Song zeigt sich, dass das Verfolgen eines Fernsehgroßereignisses - zu dem der ESC gezählt werden kann - gleichzeitig das Wissen und die Wahrnehmung über das Austragungsland, zumindest kurzfristig, beeinflusst. Die Befürchtung, dass Gastgeberländer bei Mega-Events meist von starker Kritik verschont bleiben und dadurch nur positiv dargestellt werden (Katz et al., 1981. S. 48), konnte in dieser Studie nicht bestätigt werden. Die Umsetzung der Demokratie wurde von den Vielsehern am kritischsten beurteilt. Ein Zeichen dafür, dass die Medien die Missstände aufgreifen, thematisieren und so die Schattenseiten eines Landes sichtbar machen (Giffard \& Rivenburgh, 2000). Trotzdem hat die Sendung bei den Vielsehern und Teilsehern auch positive Eindrücke hinterlassen - Aserbaidschan wurde als sehr attraktiv wahrgenommen. Somit könnte das Ziel der Eigenwerbung für sich als Reiseland und Industriestandort (Ritchie \& Smith, 1991; Florek, Breitbarth, \& Conejo, 2008; Manzenreiter, 2010) erfolgreich gewesen, die Wirkung jedoch durch die Kritik am Regierungssystem relativiert worden sein.

Auch wenn die Studie nur einen begrenzten Umfang hat, so hat sie doch einige entscheidende Erkenntnisse geliefert, an die man in zukünftigen Forschungen anknüpfen kann. So wurde der Einfluss eines Mega-Events im Fernsehen durch natürliches Nutzungsverhalten der Rezipienten offensichtlich. Durch die relative Unbekanntheit Aserbaidschans war es möglich, Medienwirkungen nachzuweisen, die sich auf einen Gegenstand beziehen, zu dem viele Menschen keine Primärerfahrungen vorweisen konnten. Für zukünftige Studien sollte jedoch ein Panel-Design angestrebt werden, um eine tatsächliche Veränderung der Bewertung (von vor der ersten ESC-Sendung bis nach dem Finale) festzustellen. Mit einer weiteren Erhebungswelle einige Wochen oder Monate nach dem Event könnte zusätzlich überprüft werden, ob und wie nachhaltig die gemessenen, kurzfristigen Lerneffekte sind. Interessant wäre das vor allem vor dem Hintergrund, dass die Berichterstattung über Austragungsländer solcher Medien-Events wieder deutlich nachlässt. In nachfolgenden Studien sollte besonders auf eine repräsentative Stichprobe Wert gelegt und auch das Instrument um einige Bewertungskategorien erweitert werden. Trotz der angesprochenen Verbesserungsmöglichkeiten hat diese Studie gezeigt, dass die Erforschung der Wirkung von Mega-Events ein großes Potential besitzt und von großer Bedeutung ist, insbesondere in Zeiten, in denen Themen zur Globalisierung und zu internationalen Beziehungen immer wichtiger werden.

\section{Literatur}

Adoni, H., \& Mane, S. (1984). Media and the Social Construction of Reality: Toward an Integration of Theory and Research. Communication Research, 11, 323-340.

Amnesty International (2012). Aserbaidschan: ESC-Organisatoren ignorieren Menschenrechtsverstöße. Abgerufen von http://www.amnesty.de/2012/5/24/aserbaidschan-escorganisatoren-ignorieren-menschenrechtsverstoesse

Arntsen, H. (2005). Staging the Nation? Nation, Myth and Cultural Stereotypes in the International Eurovision Song Contest Finals in Estonia, Latvia and Norway. In R. Bærug (Hrsg.), The Baltic media world (S. 145-157). Rīga: Flera Printing House.

Bentele, G. (1995). Der Entstehungsprozeß von Nationenimages; Informationsquellen und Verzerrungen. Überlegungen zu Grundlagen der staatlichen Auslands-Öffentlichkeits- 
arbeit. In W. A. Mahle (Hrsg.), Deutschland in der internationalen Kommunikation (S. 59-71). München: UVK-Medien, Ölschläger.

Bilandzic, H. (2006). The Perception of Distance in the Cultivation Process: A Theoretical Consideration of the Relationship Between Television Content, Processing Experience, and Perceived Distance. Communication Theory, 16, 333-355.

Custódio, M. J. F., \& Gouveia, P. M. D. C. B. (2007). Evaluation of the Cognitive Image of a Country/Destination by the Media during the Coverage of Mega-events: the Case of UEFA EURO 2004TM in Portugal. International Journal of Tourism Research, 9, 285 296.

Florek, M., Breitbarth, T., \& Conejo, F. (2008). Mega Event = Mega Impact? Travelling Fans' Experience and Perceptions of the 2006 FIFA World Cup Host Nation. Journal of Sport \& Tourism, 13, 199-219.

Gamson, W. A., Croteau, D., Hoynes, W., \& Sasson, T. (1992). Media Images and the Social Construction of Reality. Annual Review of Sociology, 18, 373-393.

Georgiou, M. (2008). "In the End, Germany will Always Resort to Hot Pants": Watching Europe Singing, Constructing the Stereotype. Popular Communication, 6, 141-154.

Gerbner, G., \& Gross, L. (1976). Living with Television. The Violence Profile. Journal of Communication, 26, 172-199.

Gerbner, G., Gross, L., Morgan, M., \& Signorielli, N. (1980). The „Mainstreaming“ of America. Violence Profile No. 11. Journal of Communication, 30, 10-29.

Gerbner, G., Gross, L., Signorielli, N., \& Morgan, M. (1980). Aging with television. Images on television drama and conceptions of social reality. Journal of Communication 30, 37-47.

Gerbner, G., Gross, L., Morgan, M., \& Signorielli, N. (1982). Charting the Mainstream. Television's Contributions to Political Orientations. Journal of Communication, 32, 100-127.

Gerbner, G., Gross, L., Morgan, M., \& Signorielli, N. (1994). Growing up with television. The cultivation perspective. In J. Bryant, \& D. Zillmann (Hrsg.), Media effects. Advances in theory and research (Lea's communication series, Aufsatzsammlung, S. 17 41). Hillsdale, NJ: Erlbaum.

Gibson, H. J., Qi, C. X., \& Zhang, J. J. (2008). Destination Image and Intent to Visit China and the 2008 Beijing Olympic Games. Journal of Sport Management, 22, 427-450.

Giffard, C. A., \& Rivenburgh, N. K. (2000). News Agencies, National Images, and Global Media Events. Journalism \& Mass Communication Quarterly, 77, 8-21.

Ginsburgh, V., \& Noury, A. G. (2008). The Eurovision Song Contest. Is voting political or cultural? European Journal of Political Economy, 24, 41-52.

Hasebrink, U., \& Schmidt, J.-H. (2012). Informationsrepertoires der deutschen Bevölkerung. Konzept für eine regelmäßig durchzuführende bevölkerungsrepräsentative Befragung im Rahmen des Vorhabens „Erfassung und Darstellung der Medien- und Meinungsvielfalt in Deutschland “. Hamburg: Hans-Bredow-Institut für Medienforschung.

Hauptmann, P., \& Lander, B. (2003). Zur Problematik von Internet-Stichproben. In A. Theobald, M. Dreyer, \& T. Starsetzki (Hrsg.), Online-Marktforschung. Theoretische Grundlagen und praktische Erfabrungen (S. 27-40). Wiesbaden: Gabler.

Hede, A. (2005). Sports-events, Tourism and Destination Marketing Strategies: an Australian Case Study of Athens 2004 and its media telecast. Journal of Sport \& Tourism, 10, 187-200. 
Hetsroni, A. (2010). When the wind changes direction: The impact of content shift on the cultivation effect. Communications, 35, 439-460.

Karimli, A. (Executive Producer). (26. Mai 2012). Eurovision Song Contest [Fernsehübertragung]. Deutschland, ARD.

Katz, E., Dayan, D., \& Motyl, P. (1981). In Defense of Media Events. In R. W. Haigh, G. Gerbner, \& R. B. Byrne (Hrsg.), Communications in the twenty-first century (S. 43-59). New York: Wiley.

Kim, S. S., \& Morrison, A. M. (2005). Change of images of South Korea among foreign tourists after the 2002 FIFA World Cup. Tourism Management, 26, 233-247.

Klenk, V. (1999). Mega-Events als Instrument der Imagepolitik. Eine Mehrmethodenstudie zu Images und Imagewirkungen der universellen Weltausstellung Expo ,92. Berlin: Vistas.

Knieper, T. (2006). Die Flut im Wohnzimmer. Publizistik, 51, 52-66.

Luhmann, N. (1996). Die Realität der Massenmedien. (2. Aufl.). Opladen: Westdeutscher Verlag.

Lippmann, W. (1929). Public Opinion. New York: The Macmillan Company.

Manzenreiter, W. (2010). The Beijing Games in the Western Imagination of China: The Weak Power of Soft Power. Journal of Sport \& Social Issues, 34, 29-48.

Martin, I. M., \& Eroglu, S. (1993). Measuring a Multi-Dimensional Construct: Country Image. Journal of Business Research, 28, 191-210.

McNelly, J. T., \& Izcaray, F. (1986). International News Exposure and Images of Nations. Journalism \& Mass Communication Quarterly, 63, 546-553.

Mende, A., Oehmichen, E., \& Schröter, C. (2012). Medienübergreifende Informationsnutzung und Informationsrepertoires. Media Perspektiven (o.Jg.), 1, 2-17.

Niedhart, G. (1996). Länderimages: Vorstellungen von anderen zwischen Selbst- und Fremdwahrnehmung. In H. Süssmuth (Hrsg.), Deutschlandbilder in Dänemark und England, in Frankreich und den Niederlanden. Dokumentation der Tagung Deutschlandbilder in Dänemark und England, in Frankreich und den Niederlanden, 15.-18. Dezember 1993, Leutherheider Forum : Historisches Seminar VII der Heinrich-HeineUniversität Düsseldorf (1. Aufl., S. 79-86). Baden-Baden: Nomos.

Pappu, R., Quester, P. G., \& Cooksey, R. W. (2007). Country Image and Consumer-Based Brand Equity: Relationships and Implications for International Marketing. Journal of International Business Studies, 38, 726-745.

Ritchie, J., \& Smith, B. H. (1991). The Impact Of A Mega-Event On Host Region Awareness: A Longitudinal Study. Journal of Travel Research, 30, 3-10.

Roche, M. (2000). Mega-events and modernity. Olympics and expos in the growth of global culture. London, New York: Routledge.

Scherer, H., \& Schlütz, D. (2003). Das inszenierte Medienereignis. Die verschiedenen Wirklichkeiten der Vorausscheidung zum European Song Contest in Hannover 2001 (1. Aufl.). Köln: von Halem.

Schweiger, W., \& Brosius, H.-B. (2003). Eurovision Song Contest - beeinflussen Nachrichtenfaktoren die Punktvergabe durch das Publikum? Medien \& Kommunikationswissenschaft, 51, 271-294.

Vallant, K. (2011). Eurovision Song Contest - Nationalismus im Wandel. In P. Pachernegg, C. Pichler, C. Pilz, D. Reicher, \& D. Semper (Hrsg.), Internationale Beziehungen aus der 
Perspektive nationaler Öffentlichkeiten. Beiträge zur Soziologie internationaler Beziebungen (1. Aufl., S. 105-122). Münster: LIT.

Weimann, G. (2000). Communicating unreality. Thousand Oaks [u.a.]: Sage Publications.

Weiss, D. P. (2008). Strategische Gestaltung des Lebenszyklus von Mega-Events (1. Aufl.). Wiesbaden: Gabler Verlag / GWV Fachverlage GmbH.

Wolther, I. (2005). Musikwettbewerb vs. Wettbewerbsmusik - Das Dilemma des Eurovision Song Contests. In D. Helms, \& T. Phleps (Hrsg.), Keiner wird gewinnen. Populäre Musik im Wettbewerb (S. 101-111). Bielefeld: Transcript.

Wolther, I. (2006). „Kampf der Kulturen“. Der Eurovision Song Contest als Mittel national-kultureller Repräsentation. Würzburg: Königshausen \& Neumann.

Wolther, I. (2008). Mehr als Musik: Die sieben Dimensionen des Eurovision Song Contests. In S. Weinacht (Hrsg.), Wissenschaftliche Perspektiven auf Musik und Medien (S. 103116). Wiesbaden: VS Verlag für Sozialwissenschaften.

Wolther, I. (2010). Musik im Medienkorsett - Der Eurovision Song Contest zwischen Kompositionswettbewerb und Fernsehereignis. In P. Moormann (Hrsg.), Musik im Fernsehen. Sendeformen und Gestaltungsprinzipien (1. Aufl., S. 11-28). Wiesbaden: VS Verlag für Sozialwissenschaften.

Yair, G. (1995). 'Unite Unite Europe' The political and cultural structures of Europe as reflected in the Eurovision Song Contest. Social Networks, 17, 147-161.

Zeng, G., Go, F., \& Kolmer, C. (2011). Beijing Olympics 2008 impact on China's image formation in international TV coverage: a media content analysis perspective. International Journal of Sports Marketing \& Sponsorship, 12, 319-336.

Zubayr, C., \& Gerhard, H. (2012). Tendenzen im Zuschauerverhalten. Media Perspektiven (o. Jg.), 3, 118-132. 


\title{
EXTENDED ABSTRACT
}

\section{The influence of televised mega-events on the perception of the hosting nation}

\author{
A study through the example of the Eurovision Song Contest in \\ Azerbaijan
}

Christiana Schallhorn

\section{The role of television in constructing reality}

Media play an important role in constructing reality (Adoni \& Mane, 1984; Gerbner \& Gross, 1976). New technologies and the expanding global networks of information and communication give media users even insights into remote places. Thus, more and more parts of the world become visible (Gamson, Croteau, Hoynes, William, \& Sasson, 1992). Especially if we are unfamiliar with, for instance, other countries and cultures, mass media exert a major influence on our perception of other nations (Bentele, 1995, S. 63). The influence of media becomes stronger the less people know about the presented issues and the less people possess personal or direct experience with the subject (Bilandzic, 2006). Television is still the most important media for catching up on the latest events in the world (Hasebrink \& Schmidt, 2012; Mende, Oehmichen, \& Schröter, 2012). This function of television as a source of socialization and cultivation has been proven many times. Specifically, it has been shown that television program featuring its homogenous messages can influence our construction of reality, and thereby, our ideas about the world (e. g. Gerbner \& Gross, 1976; Gerbner, Gross, Signorielli, \& Morgan 1980; Gerbner, Gross, Morgan, \& Signorielli, 1980, 1982, 1994). Hence, it is not surprising that, for instance, we associate the United States of America with "big cars, rock music, dancing parties" (Weimann, 2000, S. 243). Due to the repeated exposure to televised images, we take the presented information and pictures of the world for real.

Besides film productions and daily coverage about world affairs, mega events, as for example the Olympics or the FIFA World Cup, are a great opportunity for hosting countries to present themselves to a broader public (Giffard \& Rivenburgh, 2000; Ritchie \& Smith, 1991; Florek, Breitbarth, \& Conejo, 2008; Manzenreiter, 2010). Mega events can be defined as temporary big events attracting strong media attention in many countries, and thereby can reach an international audience, due to the participation of many people from different countries (modeled after Weiß, 2008; Klenk, 1999; Katz, Dayan, \& Motyl, 1981). In addition to international sporting events, the Eurovision Song Contest (ESC) can also be seen as a mega event fulfilling all mentioned criteria. It can be assumed that the coverage about a mega event also shapes the image of the hosting nation. 
Hence, viewers' perception and knowledge about the hosting country should vary depending on their amount of watching the televised event. This study examines how the broadcast of the Eurovision Song Contest 2012 in Baku and the coverage accompanying the event influence the knowledge and the perception of the hosting nation Azerbaijan. The following hypotheses and research questions are tested and answered:

H1: People having watched the show intensively (heavy-viewers) know more about Azerbaijan than people having watched the show partly (moderateviewers) or not at all (non-viewers).

RQ1: How do people possessing personal experiences with Azerbaijan or Azerbaijani differ from heavy-viewers, moderate-viewers and non-viewers possessing no such experience regarding the knowledge about Azerbaijan?

H2: People having watched the show intensively (heavy-viewers), partly (moderate-viewers) or not at all (non-viewers) differ in their perception of Azerbaijan concerning their evaluation of:

a) the attractiveness of Azerbaijan.

b) the stability of Azerbaijan.

c) the industrialization of Azerbaijan.

d) the implementation of democracy in Azerbaijan.

RQ2: How do people possessing personal experiences with Azerbaijan or Azerbaijani differ from heavy-viewers, moderate-viewers and non-viewers possessing no such experience regarding their perception of Azerbaijan as stated in $H 2$ ?

\section{Method}

An online-questionnaire was activated after the end of the show and was available online for ten days (27.05.2012-06.05.2012). The URL was published on diverse homepages and forums to call attention to the survey.

The questionnaire consisted of several parts. The first set of questions contained 17 statements about Azerbaijan (e.g. "Azerbaijan has attractive, modern cities", "Azerbaijan has a high level of industrialization" or "Azerbaijan protects the freedom of opinion"). People had to define how much they agree with these statements. A seven-point-scale was chosen (1 "not at all" to 7 "completely") to express agreement to the statements. The items can be summarized as follows: democracy $(\alpha=0.90)$, industrialization $(\alpha=0.83)$, stability $(\alpha=0.74)$ and attractiveness $(\alpha=0.89)$.

Furthermore, four knowledge-based questions were asked about Azerbaijan: "What is the capital? Who is the head of the government? What is the national language? Which country does not border Azerbaijan?" Four answer options and the option "I do not know" were given, only one answer was right.

The next part contained questions about media consumption concerning the Eurovision Song Contest (e. g. "Which parts of the Eurovision Song Contest did you watch and how much of the airtime?" Answer categories like "The performance of our singer Roman Lob", "The performance of other countries" or "the 
counting of points" could be graded from 1 "nothing at all" to 7 "completely"). Finally participants were asked if they had already been to Azerbaijan and if they knew Azerbaijani personally.

The survey was completed by 311 participants. 67 per cent were men, 33 per cent were women. The average of age was 32 years $(S D=11,1) .15$ per cent knew Azerbaijani personally and 6 per cent had already been in Azerbaijan. These people were grouped as "experienced people" $(n=52)$. Participant who watched all parts of the show as well as the preliminary decision and the semifinals were grouped as heavy viewers $(n=53)$. People who did not follow the Eurovision Song Contest at all were summarized as non-viewers $(n=45)$. And those who saw half of the show were clustered as moderate viewers $(n=52) .{ }^{12}$

\section{Results}

As shown in figure 1, almost all heavy-viewers answered all questions correctly. In contrast, non-viewers gave most seldom the right answers. That is, the more the people had watched the event, the more they knew about the country. Hypothesis 1 was supported. Research question 1, asking how people with personal experiences differ from heavy-viewers, moderate-viewers and non-viewers, can be answered as follows: People with personal experiences know as much as heavy viewers, and consequently, much more than moderate- or non-viewers.

Figure 1: Right answers given by the groups

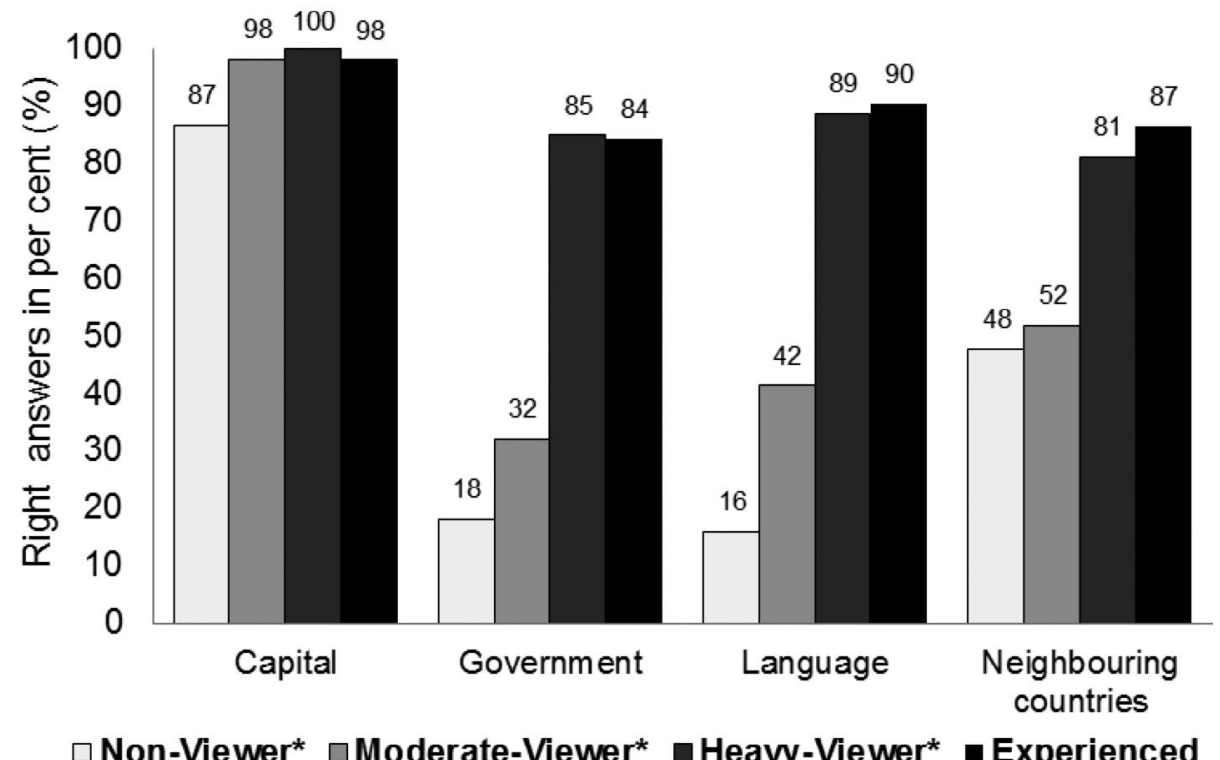

*without personal experiences with Azerbaijan/-i

12 All other participants who could not be assigned to one of the groups were excluded. 
Testing if and how the different groups vary concerning their evaluation of Azerbaijan (H2, RQ2), an analysis of variance (ANOVA) was conducted. The analysis resulted in significant effects for the evaluation of attractiveness $(F(3,199)$ $\left.=12.13, p \leq .001, \eta^{2}=.16\right)$, stability $\left(F(3,198)=3.38, p \leq .05, \eta^{2}=.05\right)$, industrialization $\left(\mathrm{F}(3,199)=7.65, p \leq .001, \eta^{2}=.10\right)$ and the implementation of democracy $\left(F(3,198)=8.14, p \leq .001, \eta^{2}=.11\right)$. Figure 2 summarizes the ANOVA results. Testing which of the viewer-groups differ significantly from the other ones, Bonferroni's post-hoc test was performed. The results indicated two interesting main findings: 1 . Only the non-viewers evaluated the attractiveness, industrialization and implementation of democracy of Azerbaijan significantly different from all other groups. 2 . The more the people watched the show, the more positive they evaluated the attractiveness and industrialization but the more negative they ranked the implementation of democracy of Azerbaijan.

\section{Figure 2: Evaluation of Azerbaijan}

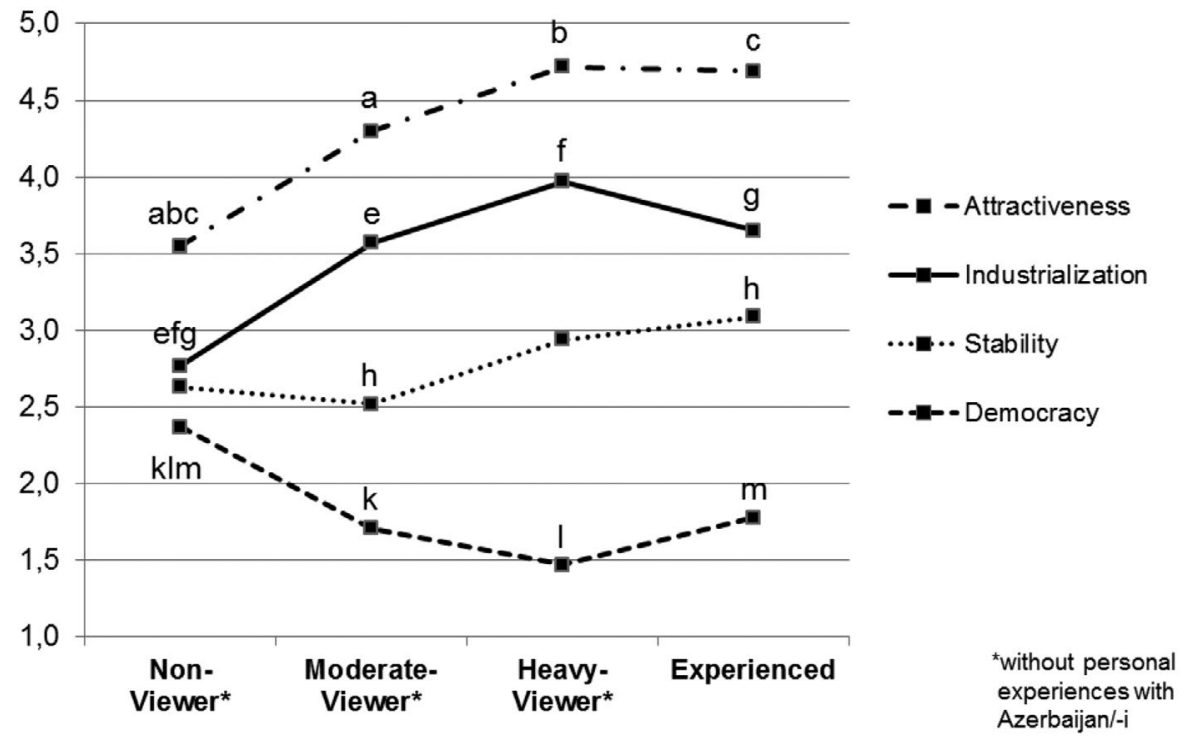

Note: Means with the same lower case differ significantly from each other. Bonferroni's post-hoc test $p \leq .05$

\section{Discussion}

The results prove not only the influence of media on perceptions of reality, but also show that even short-term events, as the Eurovision Song Contest, influence the evaluation of the hosting nation. Furthermore, the results also point to potential risks of those events, as possible grievances may become visible in public (Giffard \& Rivenburgh, 2000). In the case of the Eurovision Song Contest, the coverage about the violations of human rights led to the fact that the more the 
people had followed the show the more negatively they evaluated the implementation of democracy. In times of increasing importance of global themes this political dimension will become more important in future.

\section{References}

Adoni, H., \& Mane, S. (1984). Media and the social construction of reality: Toward an Integration of Theory and Research. Communication Research, 11, 323-340.

Bentele, G. (1995). Der Entstehungsprozeß von Nationenimages; Informationsquellen und Verzerrungen. Überlegungen zu Grundlagen der staatlichen Auslands-Öffentlichkeitsarbeit. In W. A. Mahle (Hrsg.), Deutschland in der internationalen Kommunikation (S. 59-71). München: UVK-Medien, Ölschläger.

Bilandzic, H. (2006). The Perception of Distance in the Cultivation Process: A Theoretical Consideration of the Relationship Between Television Content, Processing Experience, and Perceived Distance. Communication Theory, 16, 333-355.

Florek, M., Breitbarth, T., \& Conejo, F. (2008). Mega Event = Mega Impact? Travelling Fans' Experience and Perceptions of the 2006 FIFA World Cup Host Nation. Journal of Sport \& Tourism, 13, 199-219.

Gamson, W. A., Croteau, D., Hoynes, W., \& Sasson, T. (1992). Media Images and the Social Construction of Reality. Annual Review of Sociology, 18, 373-393.

Gerbner, G., \& Gross, L. (1976). Living with Television. The Violence Profile. Journal of Communication, 26, 172-199.

Gerbner, G., Gross, L., Morgan, M., \& Signorielli, N. (1980). The „Mainstreaming“ of America. Violence Profile No. 11. Journal of Communication, 30, 10-29.

Gerbner, G., Gross, L., Signorielli, N., \& Morgan, M. (1980). Aging with television. Images on television drama and conceptions of social reality. Journal of Communication 30, 37-47.

Gerbner, G., Gross, L., Morgan, M., \& Signorielli, N. (1982). Charting the Mainstream. Television's Contributions to Political Orientations. Journal of Communication, 32, 100-127.

Gerbner, G., Gross, L., Morgan, M., \& Signorielli, N. (1994). Growing up with television. The cultivation perspective. In J. Bryant \& D. Zillmann (Hrsg.), Media effects. Advances in theory and research (Lea's communication series, Aufsatzsammlung, S. 1741). Hillsdale, NJ: Erlbaum.

Giffard, C. A., \& Rivenburgh, N. K. (2000). News Agencies, National Images, and Global Media Events. Journalism \& Mass Communication Quarterly, 77, 8-21.

Hasebrink, U., \& Schmidt, J.-H. (2012). Informationsrepertoires der deutschen Bevölkerung. Konzept für eine regelmäßig durchzuführende bevölkerungsrepräsentative Befragung im Rabmen des Vorhabens „Erfassung und Darstellung der Medien- und Meinungsvielfalt in Deutschland“. Hamburg: Hans-Bredow-Institut für Medienforschung.

Katz, E., Dayan, D., \& Motyl, P. (1981). In Defense of Media Events. In R. W. Haigh, G. Gerbner \& R. B. Byrne (Hrsg.), Communications in the twenty-first century (S. 43-59). New York: Wiley.

Klenk, V. (1999). Mega-Events als Instrument der Imagepolitik. Eine Mehrmethodenstudie $z u$ Images und Imagewirkungen der universellen Weltausstellung Expo, 92. Berlin: Vistas. 
Manzenreiter, W. (2010). The Beijing Games in the Western Imagination of China: The Weak Power of Soft Power. Journal of Sport \& Social Issues, 34, 29-48.

Mende, A., Oehmichen, E., \& Schröter, C. (2012). Medienübergreifende Informationsnutzung und Informationsrepertoires. Media Perspektiven (o.Jg.), 1, 2-17.

Ritchie, J., \& Smith, B. H. (1991). The Impact Of A Mega-Event On Host Region Awareness: A Longitudinal Study. Journal of Travel Research, 30, 3-10.

Weimann, G. (2000). Communicating unreality. Thousand Oaks [u. a.]: Sage Publications.

Weiss, D. P. (2008). Strategische Gestaltung des Lebenszyklus von Mega-Events (1. Aufl.). Wiesbaden: Gabler Verlag / GWV Fachverlage GmbH. 